\title{
Alterações na tela de Amsler entre pacientes com catarata senil
}

\section{Changes on the Amsler grid test in patients with senile cataract}

Newton Kara-Junior ${ }^{1}$, Wilson Takashi Hida ${ }^{2}$, Marcony Santhiago $^{3}$, Iris Yamane ${ }^{3}$, Pedro Carlos Carricondo ${ }^{4}$, Newton Kara-Jose $e^{5}$

\section{Resumo}

Objetivo: Verificar a influência de alguns tipos de opacidade lenticular no teste da tela de Amsler entre portadores de catarata senil. Métodos: Foram avaliados 200 pacientes portadores de catarata senil através da aplicação da tela de Amsler, antes e após a cirurgia. O estudo foi feito em um único centro, simples cego, randomizado e prospectivo. Os pacientes foram submetidos a exame de acuidade visual (AV) corrigida, para longe e perto, biomicroscopia do segmento anterior e da região macular. Resultados: A acuidade visual média pré-operatória para longe dos 200 pacientes foi de $0,5 \log$ MAR (20/60 Tabela de Snellen). Após a cirurgia, dos 11 pacientes que haviam apresentado alteração na tela de Amsler pré-operatória, dez não tiveram alteração no exame de reavaliação da tela. Os 10 indivíduos apresentaram acuidade visual pós-operatória, com a melhor correção óptica, para distância de 20/20 e para perto de J1. No presente estudo foi constatado 5,5\% com alteração na tela de Amsler antes da cirurgia, em 5\% dos pacientes estas alterações estavam diretamente relacionados a opacidade do cristalino e $0,5 \%$ relacionados a opacidade vítrea. Conclusão: Conclui-se que a alteração no exame da tela de Amsler pode ser diretamente relacionada à catarata, na ausência de alterações maculares clínicas.

Descritores: Catarata; Extração de catarata; Facoemulsificação; Acuidade visual; Microscopia acústica/métodos

\footnotetext{
'Livre-docente, Professor Colaborador da Faculdade de Medicina da Universidade de São Paulo, Chefe do Setor de Catarata do Hospital das Clínicas da Faculdade de Medicina da Universidade de São Paulo - USP - São Paulo (SP), Brasil;

'Estagiário do Setor da Catarata da Faculdade de Medicina da Universidade de São Paulo HC-FMUSP; Assistente do Setor de Catarata da Santa Casa de Misericórdia de São Paulo;

${ }^{3}$ Estagiário do Setor da Catarata da Faculdade de Medicina da Universidade de São Paulo HC-FMUSP;

${ }^{4}$ Assistente do Setor da Catarata da Faculdade de Medicina da Universidade de São Paulo - USP - São Paulo (SP), Brasil;

${ }^{5}$ Livre-docente, Professor Titular do Departamento de Oftalmologia da Faculdade de Medicina da Universidade de São Paulo - USP São Paulo (SP); e da Faculdade de Ciências Médicas da UNICAMP - Campinas (SP), Brasil.
}

Estudo realizado no Hospital das Clínicas da Faculdade de Medicina da Universidade de São Paulo - USP - São Paulo (SP), Brasil.

Recebido para publicação em: 16/9/2008 - Aceito para publicação em 29/1/2009 


\section{INTRODUÇÃO}

A cirurgia de catarata é o procedimento mais realizado no mundo em pessoas com mais de 65 anos de idade, sendo a cirurgia que proporciona a mais eficaz recuperação funcional do órgão envolvido ${ }^{(1.3)}$. Em geral, a expectativa de pacientes que serão submetidos à facectomia é alta, fazendo-se necessária cautelosa avaliação de outras estruturas oculares a fim de estabelecer adequado prognóstico visual ${ }^{(2,3)}$.

Os avanços tecnológicos para o diagnóstico no campo da oftalmologia evoluíram nos últimos anos, porém determinados exames são caros e/ou invasivos, aumentando as dificuldades para que o paciente se submeta à cirurgia ${ }^{(1.3)}$.

O teste da tela de Amsler, introduzido em 1947 com a finalidade de avaliar alterações maculares, tem grande utilidade para detectar alterações nos 10 graus centrais da visão e sua aplicação em diferentes patologias como neurite óptica e retinopatia diabética tem se ampliado recentemente. O teste de Amsler é um exame de fácil aplicação, não invasivo e de baixo custo. Esse teste, em geral, auxilia na detecção e localização de irregularidades no campo de visão central $\left(10^{\circ}\right)$, sendo eficaz para o diagnóstico de alteração macular, que é uma importante causa de baixa visual ${ }^{(4,5)}$

No entanto, é possível que o teste com a tela de Amsler possa ser influenciado por erros refracionais e opacidades oculares, simulando alteração macular. Nos casos de DMRI, o teste contribui tanto no diagnóstico precoce como para avaliação de sua evolução. Os pacientes frequentemente levam um exemplar da tela para casa e fazem sua autoavaliação do estado da alteração macular. Não encontramos na literatura estudo relacionando a tela de Amsler com catarata senil ${ }^{(6,7)}$.

O objetivo desta pesquisa foi o de verificar a influência de alguns tipos de opacidade lenticular no teste da tela de Amsler entre portadores de catarata senil.

\section{Métodos}

Duzentos pacientes portadores de catarata senil com visão entre 0,2 e 0,7 (decimal), com melhor correção óptica na tabela de Snellen sem nenhuma doença ocular, foram selecionados para o estudo.

Na ocasião da consulta pré-operatória os indivíduos foram submetidos ao exame da tela de Amsler com a melhor acuidade visual corrigida (para perto). A tela utilizada era de cor branca com linhas pretas paralelas, horizontais e verticais, formando quadrados de $5 \mathrm{~mm}$, com a presença de um ponto central. Além de ser individual para cada paciente, para que ele pudesse desenhar o defeito do campo exatamente como via. O exame foi realizado em ambiente bem iluminado, com a tela de Amsler posicionada verticalmente a uma distância de $30 \mathrm{~cm}$ e o paciente fixando o olhar no ponto central. $\mathrm{O}$ olho contralateral foi sempre ocluído. Perguntava-se se todos os quadrados da tela eram iguais, se eram visíveis os quatro cantos do diagrama, se havia continuidade nas linhas verticais e horizontais da tela ou se existia alguma distorção. Constatado alguma alteração no exame, o paciente era solicitado a apontar e desenhar com lápis a área comprometida.

Todos os pacientes foram submetidos à cirurgia de catarata em condições pré-operatórias similares usando a mesma técnica de facoemulsificação, com o aparelho Infnity ${ }^{\oplus}$ e tecnologia NeoSonix (Alcon Laboratories, Inc). Todas as cirurgias foram realizadas por um único experiente cirurgião (NKJ). Todos pacientes foram submetidos à facoemulsificação com implante de lente intraocular AcrySof MA60AC (Alcon Laboratories,Inc). O exame da tela de Amsler foi repetido 30 dias após a realização da cirurgia.

Além da tela de Amsler foram realizados os seguintes exames: medida da acuidade visual com melhor correção óptica (para perto e para longe), biomicroscopia do segmento anterior e do vítreo e polo posterior com ênfase na macular. Para a análise dos dados, empregouse tratamento estatístico dos dados, atribuindo-se valores às categorias das variáveis.

\section{Resultados}

A acuidade visual média pré-operatória para longe dos 200 pacientes foi de $0,5 \log$ MAR (20/60 Tabela de Snellen).

$\mathrm{Na}$ ocasião do exame pré-operatório, onze pacientes apresentaram alteração no exame da tela de Amsler. A acuidade visual pré-operatória destes pacientes é apresentada nas Tabelas 1 e 2.

Após a cirurgia, dos 11 pacientes que haviam apresentado alteração na tela de Amsler pré-operatória, dez não apresentaram alteração no exame de reavaliação. Os 10 indivíduos apresentaram acuidade visual pós-operatória, com a melhor correção óptica, para distância de 20/20 e para perto de J1.

Apenas um paciente apresentou alteração no exame pré e pós-operatório, e este não manteve o mes- 
Tabela 1

Acuidade visual pré-operatória, para longe (logMAR), dos pacientes que apresentaram alteração no exame da tela de Amsler $(n=11)$

\begin{tabular}{ccc}
\hline Acuidade visual & Pacientes & \% \\
\hline 0,7 & 4 & 36.4 \\
0,5 & 4 & 36.4 \\
0,4 & 2 & 18.1 \\
0,3 & 1 & 9.1 \\
\hline
\end{tabular}

Media $=0.5(20 / 60$ Snellen $)$
Tabela 2

\section{Acuidade visual pré-operatória, para perto (Jaeger), dos pacientes que apresentaram alterações da tela de Amsler}

\begin{tabular}{ccc}
\hline Acuidade visual & Pacientes & \% \\
\hline J1 & 6 & 54.5 \\
J2 & 3 & 27.2 \\
J3 & 1 & 9.1 \\
J4 & - & - \\
J5 & 1 & 9.1 \\
\hline
\end{tabular}

Tabela 3

\section{Correlação entre a opacidade lenticular e a localização das alterações das linhas da tela de Amsler dos pacientes que apresentaram alteração ao exame*}

\begin{tabular}{|c|c|c|c|}
\hline $\mathbf{N}=\mathbf{1 1}$ & $\begin{array}{l}\text { Tipo de } \\
\text { opacidade } \\
\text { central }\end{array}$ & $\begin{array}{l}\text { Tipo e posição da } \\
\text { opacidade localizada }\end{array}$ & $\begin{array}{l}\text { Localização da } \\
\text { alteração das linhas } \\
\text { da tela de Amsler }\end{array}$ \\
\hline 1 & nuclear $1+$ & subcapsular $3+($ centro $)$ & temporal superior \\
\hline 2 & nuclear $1+$ & subcapsular $2+($ centro $)$ & nasal superior \\
\hline 3 & nuclear 1+ & subcapsular $1+($ centro $)$ & nasal superior \\
\hline 4 & nuclear 1+ & subcapsular $2+($ centro $)$, cortical anterior $2+($ centro $)$ & nasal superior \\
\hline 5 & nuclear 1+ & subcapsular $1+($ centro $)$, cortical anterior $1+($ centro $)$ & nasal superior \\
\hline 6 & nuclear $1+$ & subcapsular posterior $1+($ temporal superior $)$ & nasal superior \\
\hline 7 & nuclear $2+$ & subcapsular posterior $1+($ centro $)$ & temporal inferior \\
\hline 8 & nuclear $1+$ & subcapsular $1+$ (difusa), cortical anterior (temporal inferior). & todos os quadrantes \\
\hline 9 & nuclear $1+$ & $\begin{array}{l}\text { cortical anterior } 2+\text { (nasal e temporal inferior), cortical posterior } \\
\text { (nasal e temporal inferior) }\end{array}$ & nasal superior \\
\hline
\end{tabular}

*segundo sistema de classificação para opacidade de lente (LOCS II)

mo padrão de alteração nas linhas da tela em ambas oportunidades. O teste foi repetido após este indivíduo movimentar a cabeça, sendo observado que a região comprometida mudava de posição. $\mathrm{O}$ exame de biomicroscopia macular e a tomografia de coerência óptica, não mostraram alterações. Neste paciente, acuidade visual, com a melhor correção óptica, para distância foi de 20/20 e para perto de J1.

A correlação entre a opacidade lenticular e a localização das alterações das linhas da tela de Amsler dos pacientes que apresentaram alteração no exame da tela é apresentada na Tabela 3.

\section{Discussão}

A alta expectativa de reabilitação visual em pacientes submetidos a cirurgia de catarata torna importante a orientação dos mesmos a respeito do prognóstico visual
${ }^{(8)}$. Quando existe suspeita de que a o pacidade de cristalino não justifica a baixa de visão e o paciente apresenta alguma alteração macular à fundoscopia, são necessários testes adicionais para avaliar a integridade macular ${ }^{(9)}$.

A angiografia retiniana com fluoresceína e a tomografia de coerência óptica OCT fornecem suporte diagnóstico que complementam as impressões clínicas ${ }^{(10,11)}$. Porém, tratam-se de exames nos quais o paciente pode ter reações adversas ao contraste, ou é de custo relativamente elevado e pode ser de difícil acesso ${ }^{(12-14)}$.

O teste de Amsler é de fácil aplicação, baixo custo e universalmente disponível, sendo útil nos exames oftalmológicos de rotina ${ }^{(15)}$. Por esta razão, deve-se indicar com freqUência uma autoavaliação com o teste de Amsler nos pacientes portadores de catarata, ou orientar quanto às queixas de metamorfopsias, escotoma, visão embaçada e micropsias. Mesmo tendo limitações ${ }^{(11)}$, tem sido considerado um exame prévio indispensável 
para o diagnostico de defeitos de campo visual ${ }^{(11,16-18)}$.

Outros autores ${ }^{(6,7)}$ demonstraram que opacidades lenticulares localizadas podem estar associadas à alteração do exame de tela de Amsler, podendo comprometer a precisão da avaliação macular na ocasião do exame pré-operatório para cirurgia de catarata.

O estudo sugere que opacidades lenticulares podem estar relacionadas a alterações no teste da tela de Amsler simulando alterações maculares. Ao se tentar associar à posição da opacidade lenticular localizada com as linhas da tela de Amsler não se encontra correlação (Tabela 3). No único caso em que a alteração na tela de Amsler se manteve, suspeita-se de que esta se justifique por condensações vítreas importantes. Considerase, portanto, que mais estudos nessa linha devam ser realizados.

Este estudo sugere haver contribuição da catarata em testes de função macular, já que as alterações da tela de Amsler desapareceram em 91\% dos casos após a facectomia.

\section{Abstract}

Purpose: To assess the influence of some forms of lenticular opacification in the Amsler grid test among patients suffering from senile cataract. Methods: Randomized, prospective interventional trial. Two hundred patients with senile cataract were evaluated with Amsler grid, measurement of best corrected visual acuity for near distance and far distance, biomicroscopy of the anterior segment and macular region. Results: With a two months minimum of follow-up, the average visual acuity after surgery for far distance among the 200 patients was 0.48 $\log M A R, 11(5.5 \%)$ had changes in the Amsler grid test before surgery. After surgery, from the 11 subjects with changes in the preoperative Amsler grid test, 10 had no more changes in the Amsler grid. These 10 subjects had postoperative best corrected visual acuity of 20/20, and Jaeger 1, no abnormalities of the fundus having been detected. Under this study conditions, the prevalence of changes in the Amsler grid test was $5.5 \%$, and in $5 \%$ of the patients these changes were likely related to cataract whereas in $0.5 \%$ they were related to vitreous opacification. Conclusion: Some patient with cataract showed changes in the Amsler grid test that can be directly related to cataract, in the absence of clinically detected macular disorders.

Keywords: Cataract; Cataract extraction; Phacoemulsification; Visual acuity; Microscopy, acoustic / methods

\section{REFERÊNCIAS}

1. José NK, Delgado AMN, Arieta CEL. Exeqüibilidade da cirurgia de catarata em hospital-escola: em busca de um modelo econômico. Rev Assoc Med Bras (1992). 1994;40(3):186-8.

2. Thylefors B. Much blindness is avoidable. World Health Forum. 1991;12(1):78-86.

3. Thylefors B. Avoidable blindness. Bull World Health Organ. 1999;77(6):453.

4. Kara-Júnior N, Temporini ER, Kara-José N. Cataract surgery: expectations of patients assisted during a community project in Sao Paulo, state of São Paulo, Brazil. Rev Hosp Clin Fac Med Univ São Paulo. 2001;56(6):163-8.

5. Wall M, Sadun AA. Threshold Amsler grid testing. Crosspolarizing lenses enhance yield. Arch Ophthalmol. 1986;104(4):520-3.

6. Bernth-Petersen P. Evaluation of the transilluminated Amsler grid for macula testing in cataract patients. Acta Ophthalmol (Copenh). 1981;59(1):57-63.

7. Miller D, Lamberts DW, Perry HD. An illuminated grid for macular testing. Arch Ophthalmol. 1978;96(5):901-2.

8. Tranos PG, Ghazi-Nouri SM, Rubin GS, Adams ZC, Charteris DG. Visual function and subjective perception of visual ability after macular hole surgery. Am J Ophthalmol. 2004;138(6):995-1002.

9. Gibson JM, Shaw DE, Rosenthal AR. Senile cataract and senile macular degeneration: an investigation into possible risk factors. Trans Ophthalmol Soc U K. 1986;105( Pt 4):463-8.

10. Indocyanine green angiography. American Academy of Ophthalmology. Ophthalmology. 1998;105(8):1564-9.

11. Achard OA, Safran AB, Duret FC, Ragama E. Role of the completion phenomenon in the evaluation of Amsler grid results. Am J Ophthalmol. 1995;120(3):322-9.

12. Friedman SM, Margo CE. Choroidal neovascular membranes: reproducibility of angiographic interpretation. Am J Ophthalmol. 2000;130(6):839-41.

13. Klein R, Klein BE, Linton KL. Prevalence of age-related maculopathy. The Beaver Dam Eye Study. Ophthalmology. 1992;99(6):933-43.

14. Vinding T. Visual impairment of age-related macular degeneration. An epidemiological study of 1000 aged individuals. Acta Ophthalmol (Copenh). 1990;68(2):162-7.

15. Yannuzzi LA, Rohrer KT, Tindel LJ, Sobel RS, Costanza MA, Shields W, Zang E. Fluorescein angiography complication survey. Ophthalmology. 1986;93(5):611-7.

16. Fine AM, Elman MJ, Ebert JE, Prestia PA, Starr JS, Fine SL. Earliest symptoms caused by neovascular membranes in the macula. Arch Ophthalmol. 1986;104(4):513-4.

17. Roy MS. Vision loss without Amsler grid abnormalities in macular subretinal neovascularization. Ophthalmologica. 1985;191(4):215-7.

18. Schuchard RA. Validity and interpretation of Amsler grid reports. Arch Ophthalmol. 1993;111(6):776-80.

\section{ENDEREÇO PARA CORRESPONDÊNCIA: \\ Rua Teodoro Sampaio, $n^{\circ} 498$ - apto. 35 - Pinheiros \\ CEP 05406-000 - São Paulo - SP \\ E mail: marconysanthiago@hotmail.com}

